\title{
A NOVEL HYBRID NODE LOCALIZATION ALGORITHM FOR WIRELESS SENSOR NETWORKS
}

\author{
${ }^{1}$ Liu Yu, ${ }^{1}$ Wang Haipeng, ${ }^{1}$ He You and ${ }^{2}$ Shen Jian \\ ${ }^{1}$ Research Institute of Information Fusion, \\ ${ }^{2}$ Training centre of New Equipment, \\ Naval Aeronautical and Astronautical University, Yantai 264001, China
}

Received 2013-08-21, Revised 2013-09-04; Accepted 2013-10-29

\begin{abstract}
Node self-localization is one of the supporting technologies in Wireless Sensor Networks (WSNs). In this study, a distributed hybrid localization algorithm based on divide-and-conquer and refinement method is proposed. Firstly, the effect of ranging error in the Received Signal Strength (RSS) model on node localization is analyzed and the topology relations of anchors and unknown nodes are derived when the least localization error is achieved and then, the localization-union scheme is designed and refinement method is presented to improve the estimate accuracy. Finally, the simulation results prove that our proposed algorithm has the merits of superior performance, simple design and less overhead. Especially, it can use sparse anchors to achieve high localization accuracy.
\end{abstract}

Keywords:Wireless Sensor Networks, Node Self-localization, Divide-And-Conquer Algorithm, Localization-Union, Weighted Refinement

\section{INTRODUCTION}

Wireless Sensor Networks (WSNs) are made up of small devices (nodes) which are capable of collecting information from the environment such as temperature, vibrations, humidity, sound, light and motions. A WSN can be used in military surveillance, environmental monitoring, intelligent spaces, habitat and structural monitoring and robotics, among others. A WSN can be also used for an early detection of a forest fire (Wang et al., 2005), in doing so the damaged area could be considerably reduced and the wildlife also could be preserved. In critical applications, we would like to know as soon as possible the geographical node position that reports the incident. Nowadays, there are many Node Localization Algorithms (NLAs) with different accuracies and approaches. Despite the large amount of NLAs, they can be classified into two main groups: range-free and range-based NLAs. In the former, NLAs use only the content of received messages; a good example of this classification is the Centroid Localization (CL) (Bulusu et al., 2000). In the latter, NLAs utilize distances or angles; e.g., RADAR and the GPS. There are also NLAs that belong to both classes because they use the content of the received messages and distances/angles. These are referred to as hybrid NLAs; e.g., the Triangular Centroid Localization Algorithm (Jauregui-Ortiz et al., 2011), Weighted Centroid Localization (Blumenthal et al., 2007), Sequential Greedy Optimization based Localization Algorithm (Shi et al., 2010), Weighted centroid correction localization (Li et al., 2011) and Improved Centroid Localization (Liu et al., 2012). However, the integration of a large amount of factors into a NLA is not related directly to a high accuracy. In fact, the integration of all possible and suitable factors for a given real environment into a single NLA might be not feasible to achieve. In some cases, NLAs are designed for specific scenarios and only the most Corresponding Author: Liu Yu, Research Institute of Information Fusion, Naval Aeronautical and Astronautical University, Yantai 264001, China 
representative factors are considered (Barani and Fathy, 2010; Iskandarani, 2011; Newlin and Monie, 2013).

As we all know that there is not a universal localization algorithm that presents the same accuracy for all possible scenarios. Based on the analyses of existing localization algorithms and the application of WSNs, we can conclude that a perfect localization scheme for large-scale networks have to satisfy these merits as follows. (1) High localization accuracy; (2) Self-configuration; (3) decentralized localization; (4) Low communication and computation cost; (5) Robustness. All of this leads us to propose a novel hybrid node localization algorithm named Divide-andconquer and Refinement Method Based Localization Algorithm (DRBLA) for WSNs.

\subsection{Related Work}

Hereafter, the term anchors refers to those nodes for which their location is known and UNs (UNs) for those nodes for which their location is unknown. The next subsections describe several representative hybrid NLAs.

\subsubsection{Hybrid NLAs}

Hybrid NLAs use both the content of the received messages and distances/angles. Some of these algorithms include RSSI maximum likelihood estimation scheme (RSSI-MLE) (Liu, 2009), the Weighted Centroid Localization (WCL) (Jauregui-Ortiz et al., 2011), Improved Centroid Localization Algorithm (ICL) (Liu et al., 2012) and EDIPS (Vera et al., 2011). The ICL algorithm is based on APIT (He et al., 2003) and the quality of perpendicular bisector. Three perpendicular bisectors divide the beacon triangle formed by the APIT algorithm into six small cells. An UN assigns itself to a cell by the RSSI from heard beacon nodes. Finally, the centroid of the assigned cell is assumed as the position of the UN. The EDIPS algorithm matches with both hybrid NLAs and context-aware applications because it uses the signal strength from reference points and reacts regarding the environment status.

\subsubsection{Analysis of RSSI-MLE Algorithm}

In RSSI localization scheme, emission signal strength of emission nodes is known, receiving nodes calculate the signal transmission loss with receiving signal strength, theory and empirical model is used to transform transmission loss into distance, then existing algorithms are used to calculate location of the node.
When UN receives emission signal of three anchors, triangular centroid localization is used to calculate selfcoordinate. If more than three anchors are received, maximum likelihood estimation can be used to locate. In this study, this RSSI maximum likelihood estimation scheme is called RSSI-MLE localization scheme.

RSSI-MLE algorithm takes the influence of the distance of anchors and UNs into consideration and high localization accuracy is achieved with accurate distance (Amitangshu, 2010). However, RSSI location algorithm has the characteristic that close distance is relatively accurate and far distance is relatively fuzzy, in addition, topology structure of the anchors has a certain influence on localization of UNs, so RSSIMLE algorithm do not take full advantage of redundant information of the networks, the effect is general in practical application.

\subsubsection{Analysis of Weighed Centroid Algorithm}

With the analysis of radio transmission loss model, (He et al., 2003) finds that simple centroid algorithms do not reflect the influence of different anchors and then reduce the accuracy, so $\mathrm{W}$-Centroid is developed, in which the weighing factor is used to indicate the influence of anchors. The formula that W-Centroid calculates coordinate of $\mathrm{UN}\left(\mathrm{x}_{\mathrm{i}}, \mathrm{y}_{\mathrm{i}}\right)$ is Equation (1):

$$
\begin{aligned}
& \left(x_{i}, y_{i}\right)=\left\{\begin{array}{l}
\frac{x_{1}}{d_{1}+d_{2}}+\frac{x_{2}}{d_{2}+d_{3}}+\frac{x_{3}}{d_{3}+d_{1}} \\
\frac{1}{d_{1}+d_{2}}+\frac{1}{d_{2}+d_{3}}+\frac{1}{d_{3}+d_{1}}
\end{array}\right. \\
& \left.\frac{\frac{\mathrm{y}_{1}}{\mathrm{~d}_{1}+\mathrm{d}_{2}}+\frac{\mathrm{y}_{2}}{\mathrm{~d}_{2}+\mathrm{d}_{3}}+\frac{\mathrm{y}_{3}}{\mathrm{~d}_{3}+\mathrm{d}_{1}}}{\frac{1}{\mathrm{~d}_{1}+\mathrm{d}_{2}}+\frac{1}{\mathrm{~d}_{2}+\mathrm{d}_{3}}+\frac{1}{\mathrm{~d}_{3}+\mathrm{d}_{1}}}\right\}
\end{aligned}
$$

where, $\left(\mathrm{x}_{1}, \mathrm{y}_{1}\right),\left(\mathrm{x}_{2}, \mathrm{y}_{2}\right)$ and $\left(\mathrm{x}_{3}, \mathrm{y}_{3}\right)$ are coordinates of three anchors, $d_{1}, d_{2}$ and $d_{3}$ are the distance between the three anchors and the UN respectively. The weights indicate that closer anchor has lager influence on the coordinate of UN, thus localization accuracy is improved with this internal relation.

W-Centroid translates range information into weighing factors and greatly improves accuracy in contrast to centroid localization. But W-Centroid do not resolve bugs in anisotropic networks, in other words, irregular distribution of nodes in networks still has a great influence on W-Centroid. 


\subsection{DRBLA}

There are three main stages in DRBLA. Firstly, distances between nodes are measured. Secondly, relative angles between anchors and UN are estimated and effective verifying node is selected out to identify estimate coordinate. Finally, the weighted centroid of all the cursory coordinates is identified as the possible location of UN.

Information about distance between nodes in network is used to localize UNs in DRBLA. Firstly, localization information is broadcasted from all anchors and thus distance between anchors and UNs can be calculated out based on the RSS received by UNs. Then a virtual polar coordinate system is established for location calculation and some special anchors are selected as localization union, which is shown in Fig. 1. In this way, one or two estimate position of a UN can be achieved. Then, another suitable anchor is taken as a verifier to find the most likely location of the UN. Finally, the resulting location of the UN is refined by the weighted distance between anchors and the UN.

\subsubsection{Nodes RSS Ranging Model}

When some actual factors such as multipath, reflection and obstacle are considered, signal propagation is always anisotropic and path attenuation varies in comparison with theoretical value. However, log-normal distribution model introduces environment noise into the calculation of RSS, which is closer to the actual application and is shown as follows Equation (2):

$$
\left[\frac{\mathrm{P}_{\mathrm{r}}(\mathrm{d})}{\mathrm{P}_{\mathrm{r}}\left(\mathrm{d}_{0}\right)}\right]_{\mathrm{dB}}=-10 \mathrm{n} \times \lg \left[\frac{\mathrm{d}}{\mathrm{d}_{0}}\right]+\mathrm{X}_{\mathrm{dB}}
$$

where, $\mathrm{d}_{0}$ represents the reference distance and generally is set to be $1 ; \mathrm{P}_{\mathrm{r}}\left(\mathrm{d}_{0}\right)$ denotes the received signal power at $\mathrm{d}_{0} ; \mathrm{X}_{\mathrm{dB}} \sim \mathrm{N}(0, \sigma)$ represents the existing Gaussian random variable and $4 \leq \sigma \leq 10$ usually; $\mathrm{n}$ denotes the signal attenuation factor and $2 \leq n \leq 5$. As is shown in (3), if environment noise is not considered, received signal power is a function which is monotone decreasing with distance. Assume that Equation (3 and 4):

$$
\begin{aligned}
& A=P_{r}\left(d_{0}\right)-X_{d B} \\
& R S S(d)=10 \lg \left(P_{r}(d)\right)
\end{aligned}
$$

Then we can rewrite (2) as Equation (5):

$$
\operatorname{RSS}(\mathrm{d})=\mathrm{A}-10 \mathrm{n} \times \lg (\mathrm{d})
$$

Wireless signal received strength exists determinate logarithmic relationship with propagation distance. As is shown in Fig. 2, when propagation distance is short, signal attenuation is very fast, but distance is not very sensitive to the change of received signal strength.

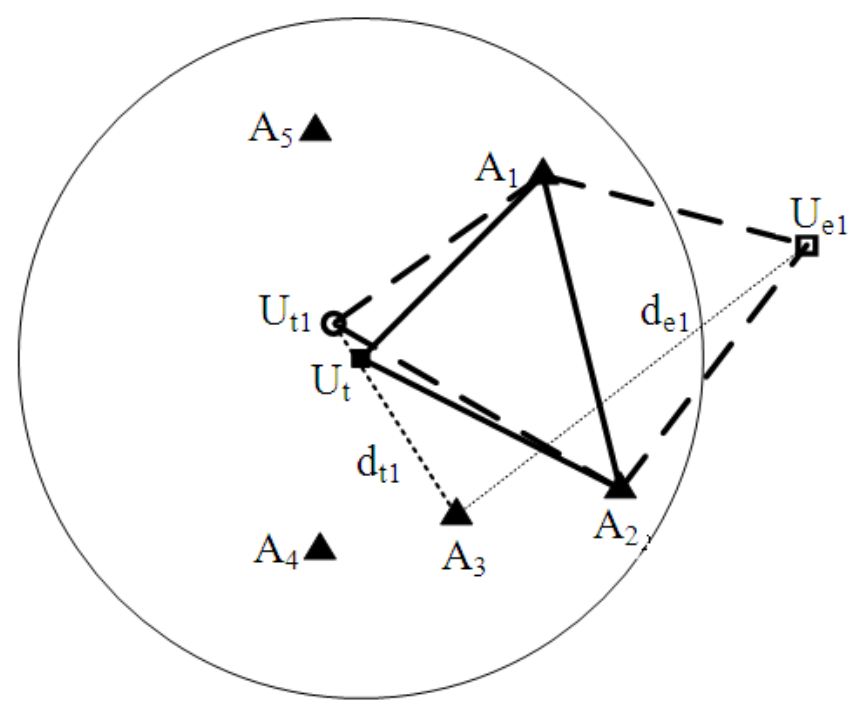

-Anchor - UN • Estimate location - Pseudo location

Fig. 1. DRBLA overview 


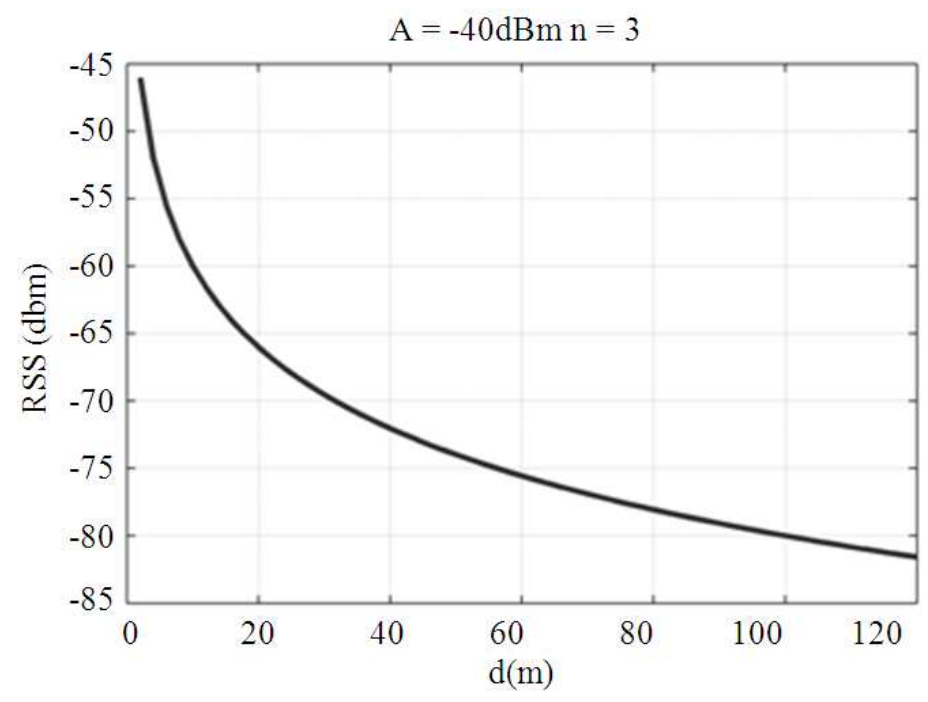

Fig. 2. RSS varying range in perfect circumstance

When propagation distance is very long, signal attenuation is close to linear and a slight change of received signal strength will lead to great diversity for distance estimation. Consequently it can be seen that RSSI which measures distance through signal attenuation has the characteristic that close distance is relatively accurate and far distance is relatively fuzzy.

\subsubsection{Analyses of Localization Based on RSS}

If there does not exist range error between nodes, a UN can be exactly localized by three anchors in twodimension networks. In the same way, we can achieve two estimate position of a UN using two anchors (anchor-union) and it is not difficult to get rid of the wrong position by using another suitable anchor, which is named Verified Node (VD). Assume that the location of the anchor-union is $A_{i}\left(x_{i}, y_{i}\right)(i=1,2)$ and the position of $\mathrm{UN}$ is $\mathrm{U}\left(\mathrm{x}_{\mathrm{e}}, \mathrm{y}_{\mathrm{e}}\right)$, the distance between each anchor and the UN can be denoted by $d_{i}(i=1$, 2). Then, we have Equation (6):

$$
\left\{\begin{array}{l}
\left(x_{e}-x_{1}\right)^{2}+\left(y_{e}-y_{1}\right)^{2}=d_{1}^{2} \\
\left(x_{e}-x_{2}\right)^{2}+\left(y_{e}-y_{2}\right)^{2}=d_{2}^{2}
\end{array}\right.
$$

There are three possible results of solving the above equations, two solutions, one solution and no solution, respectively. To guarantee that there is solution for the equation, we can derive that some condition must be satisfied as follows Equation (7):

$$
\left\{\begin{array}{l}
\left|\mathrm{d}_{1}+\mathrm{d}_{2}\right| \geq \sqrt{\left(\mathrm{x}_{1}-\mathrm{x}_{2}\right)^{2}+\left(\mathrm{y}_{1}-\mathrm{y}_{2}\right)^{2}} \\
\left|\mathrm{~d}_{1}-\mathrm{d}_{2}\right| \leq \sqrt{\left(\mathrm{x}_{1}-\mathrm{x}_{2}\right)^{2}+\left(\mathrm{y}_{1}-\mathrm{y}_{2}\right)^{2}}
\end{array}\right.
$$

The result of two solutions will be rightly discussed in the following part. In this situation, there are two points of intersection formed by the two cycles, which are established by the position of anchors (A1 and A2) and distance ( $\mathrm{d} 1$ and $\mathrm{d} 2$ ) between anchors and the $\mathrm{UN}$, as shown in Fig. 3.

When there exists range error of $\mathrm{d}_{\mathrm{i}}$, the solution of (6) will not be precise enough. The range error of RSS range model is supposed to be $\varepsilon>0$, the range value will be in $\left(d_{i}-\varepsilon, d_{i}+\varepsilon\right)$ if the actual distance between two nodes. For the reason that sensor nodes are densely deposited and the communication range is very short, the range error is assumed to be identical in this study. Then, the analysis of two-node localization in the actual environment is shown in Fig. 4.

As shown in Fig. 4, the intersection area formed by the four cycles denotes the localization error and it can be labeled by $C_{U}$ without loss of generality. $S_{U}$ represents a circle whose circle is $U$ and radius is $\varepsilon$. A line through $U$ and $A_{i}$ has two intersections with borderline of $\mathrm{S}_{\mathrm{U}}$, through which tangent lines are drawn, so intersection points of tangent lines are A, B, $\mathrm{C}$ and $\mathrm{D}$, which consists of diamond $\mathrm{ABCD}$ as shown in Fig. 5. 


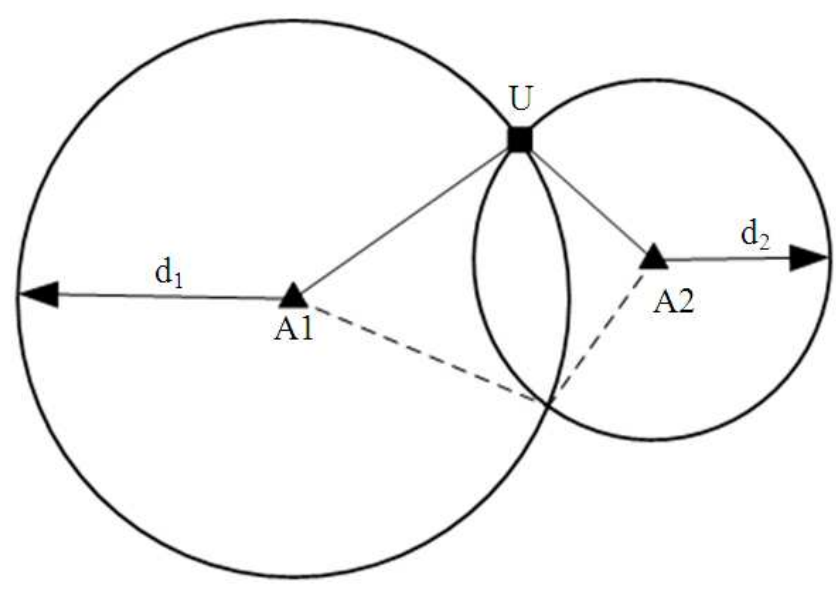

Anchor

UN

Fig. 3. Two-nodes localization model

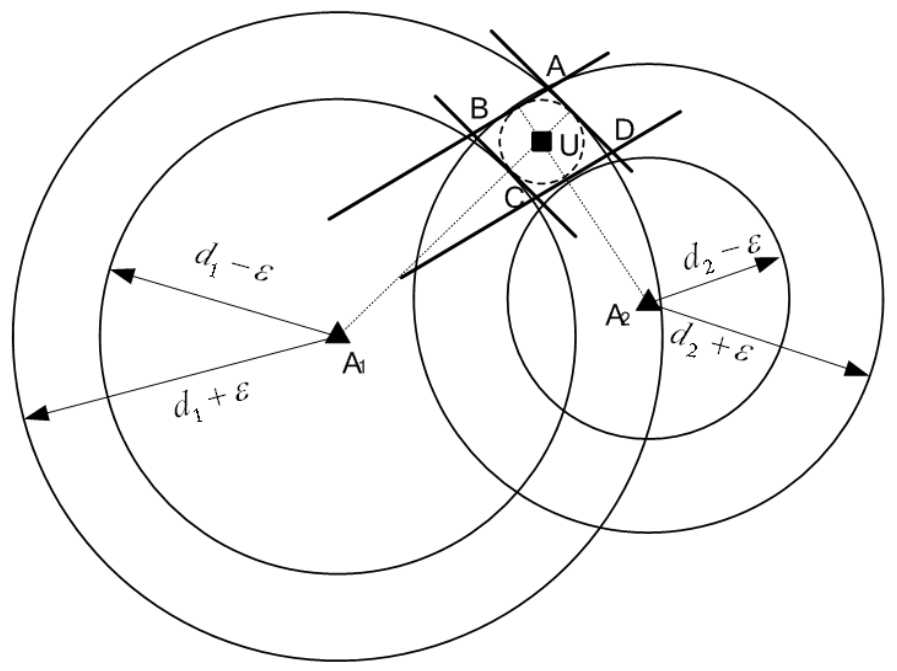

Fig. 4. Two-nodes localization with range error

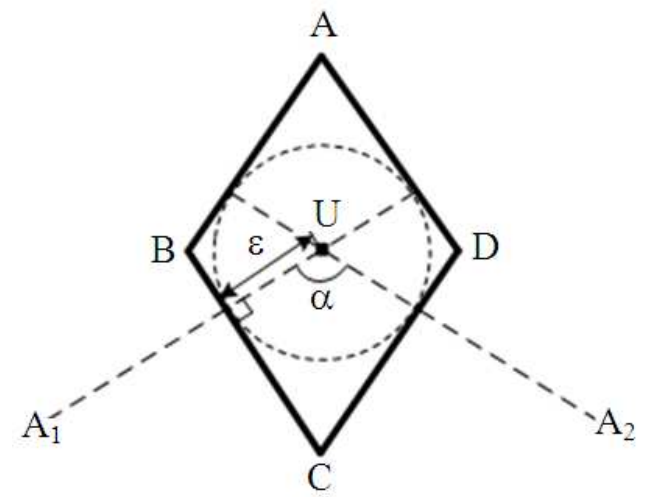

Fig. 5. Localization error area

When $\varepsilon$ is small enough, border of $\mathrm{C}_{\mathrm{U}}$ can be lined, then the area $\left(\tilde{C}_{U}\right)$ of diamond $\mathrm{ABCD}$ can be seen as the agonic estimation of $\mathrm{C}_{\mathrm{U}}$. In this way, estimation of ranging error is transformed into the estimation of the area of diamond ABCD. The problem how to get the least localization error will be discussed as follows.

Zhou (2009) has shown these following remarks.

\section{Definition 1:}

A subset $T \in \mathrm{R}^{\mathrm{n}}$ is called convex set if $\left(\lambda_{\mathrm{x} 1}+(1-\lambda) \mathrm{x}_{2}\right)$ $\in \mathrm{T}$ where $\lambda \in[0,1]$ denotes the random real number and $\mathrm{x}_{1}, \mathrm{x}_{2} \in \mathrm{T}$ represent two random nodes. 


\section{Definition 2:}

A function $f(x)$ is called strict increasing function in $\mathrm{T}$ if the following condition can be satisfied. $\mathrm{F}\left(\lambda_{\mathrm{x} 1}+(1-\right.$ $\left.\lambda) \mathrm{x}_{2}\right) \leq \mathrm{f}\left(\mathrm{x}_{1}\right)+(1-\lambda) \mathrm{f}\left(\mathrm{x}_{2}\right)$.

\section{Lemma 1:}

For a smooth function $f(x)$ defined in subset $T \in R^{n}$ and $f(x)$ is second-order continuous differentiable, if Hesse matrix of $f(x)$ is positive definite everywhere then $f(x)$ is a strict convex function in $T$.

\section{Lemma 2:}

For a convex function $f(x)$ defined in subset $T \epsilon$ $\mathrm{R}^{\mathrm{n}}$, there is:

$$
f\left(\frac{1}{m}\left(x_{1}+x_{2}+L+x_{m}\right)\right) \leq \frac{1}{m} g\left(f\left(x_{1}\right)+f\left(x_{2}\right)+L+f\left(x_{m}\right)\right)
$$

where, $\mathrm{x}_{1}, \mathrm{x}_{2}, \ldots \mathrm{x}_{\mathrm{m}}$ represents $\mathrm{m}$ points in the subset; And if $f(x)$ is strict convex function, the in Equation (8) can achieved equation only when $\mathrm{x}_{1}=\mathrm{x}_{2}=\ldots=\mathrm{x}_{\mathrm{m}}$.

Based on the above analyses, we can derive out a theorem as follows.

\section{Theorem 1:}

If a $\mathrm{UN} \mathrm{U}\left(\mathrm{x}_{\mathrm{e}}, \mathrm{y}_{\mathrm{e}}\right)$ is to be localized by a anchor-union $A_{i}\left(x_{i}, y_{i}\right)$, the least localization error can be achieved when the angle formed by $U$ and $A_{i}$ is $\pi / 2$.

\section{Proof:}

$\tilde{C}_{U}$ can be considered as the external diamond $\mathrm{ABCD}$ as shown in Fig. 5, then we have Equation (9):

$$
\mathrm{S}\left(\tilde{\mathrm{C}}_{\mathrm{U}}\right)=2 \varepsilon^{2}\left(\tan \frac{\alpha}{2}+\tan \frac{\pi-\alpha}{2}\right)
$$

And also we have $\alpha+(\pi-\alpha)=\pi$ and $(\tan \alpha)^{\prime \prime}=2 \tan$ $\alpha(1+\tan \alpha) \geq 0$, then the following derivation can be achieved based on lemma 1 and lemma 2 when $0 \leq \alpha \leq \pi / 2$ Equation (10):

$$
\begin{aligned}
& \mathrm{S}\left(\tilde{\mathrm{C}}_{\mathrm{U}}\right)=4 \varepsilon^{2} \times \frac{1}{2}\left(\tan \frac{\alpha}{2}+\tan \frac{\pi-\alpha}{2}\right)^{3} \\
& 4 \varepsilon^{2} \times \tan \frac{\alpha+(\pi-\alpha)}{4}=4 \varepsilon^{2} \times \tan \frac{\pi}{4}
\end{aligned}
$$

When $\alpha=\pi-\alpha$ which means $\alpha=\pi / 2$, the equation can be achieved. In other words, when angle formed by two anchors and the $\mathrm{UN}$ is $\pi / 2$, the least estimate error can be achieved.
Because Equation (9) is strict convex function, the resulting extremum is the one and only solution, when angle between two anchors and UN is close to $\pi / 2$, the function value is smaller. In actual application, a reasonable $\lambda_{\alpha}$ can be chosen with the density of anchors and anchors can be used as localization-union only if $\pi / 2-\lambda_{\alpha} \leq \alpha \leq \pi / 2+\lambda_{\alpha}$.

However, for Equation (6), there may be two solutions, one of which should be got rid of. In DRBLA, the real estimation location of $U$ will be verified by another redundant anchor which will be specified as follows.

If anchors $A_{i}$ and $A_{i+1}$ are used as a localizationunion, two estimate location $\mathrm{U}_{\mathrm{ei}}\left(\mathrm{x}_{\mathrm{ei}}, \mathrm{y}_{\mathrm{ei}}\right)$ and $\mathrm{U}_{\mathrm{ti}}\left(\mathrm{x}_{\mathrm{ti}}, \mathrm{y}_{\mathrm{ti}}\right)$ are obtained from Equation (4-6). Then another anchor $A_{j}\left(x_{j}, y_{j}\right)$ is employed to validate the real position of $U N$. Let $d_{e i}$ and $d_{t i}$ denote the distance between $U_{e i}$ and $A_{j}$, $\mathrm{U}_{\mathrm{ti}}$ and $\mathrm{A}_{\mathrm{j}}$, respectively. We have Equation (11):

$\left\{\begin{array}{l}\mathrm{d}_{\mathrm{ei}}^{\prime}=\sqrt{\left(\mathrm{x}_{\mathrm{ei}}-\mathrm{x}_{\mathrm{j}}\right)^{2}+\left(\mathrm{y}_{\mathrm{ei}}-\mathrm{y}_{\mathrm{j}}\right)^{2}} \\ \mathrm{~d}_{\mathrm{ti}}^{\prime}=\sqrt{\left(\mathrm{x}_{\mathrm{ti}}-\mathrm{x}_{\mathrm{j}}\right)^{2}+\left(\mathrm{y}_{\mathrm{ti}}-\mathrm{y}_{\mathrm{j}}\right)^{2}}\end{array}\right.$

The error of $d_{e i}^{\prime}, d_{t i}^{\prime}$ to the actual range $d_{j}$, which is computed by RSS ranging model, can be calculated by Equation (12):

$\left\{\begin{array}{l}\operatorname{Err}_{\mathrm{ei}}=\left\|\mathrm{d}_{\mathrm{ei}}^{\prime}-\mathrm{d}_{\mathrm{j}}\right\| \\ \operatorname{Err}_{\mathrm{ti}}=\left\|\mathrm{d}_{\mathrm{ti}}^{\prime}-\mathrm{d}_{\mathrm{j}}\right\|\end{array}\right.$

Then $\operatorname{Err}_{\mathrm{ei}}$ is compared with $\mathrm{Err}_{\mathrm{ti}}$ and we obtain the smaller one which is corresponding to the real estimate location of the UN.

For clarity, anchors $\mathrm{A}_{1}$ and $\mathrm{A}_{2}$ are localization union and $\mathrm{U}_{\mathrm{e} 1}\left(\mathrm{x}_{\mathrm{e} 1}, \mathrm{y}_{\mathrm{e} 1}\right), \mathrm{U}_{\mathrm{t} 1}\left(\mathrm{x}_{\mathrm{t} 1}, \mathrm{y}_{\mathrm{t} 1}\right)$ are the two estimate position which should be verified as shown in Fig. 1. Here, another anchor $\mathrm{A}_{3}$ is chosen to be a verifier and the distance between this verifier and the two estimate position are calculated and labeled as $\mathrm{d}_{\mathrm{e} 1}^{\prime}$ and $\mathrm{d}_{\mathrm{t} 1}$. Finally, we can conclude that $\mathrm{U}_{\mathrm{t} 1}\left(\mathrm{x}_{\mathrm{t}}, \mathrm{y}_{\mathrm{t} 1}\right)$ is the real estimate position of the $\mathrm{UN}$ after we get $\operatorname{Err}_{\mathrm{t} 1}<\mathrm{Err}_{\mathrm{e} 1}$.

Generally, the unsuitable one of two solutions of Equation (6) can be get rid of by the above verifying method. However, this method won't make it if the verifier is almost in line with the localization-union.

As shown in Fig. 6, $A_{3}$ and $A_{4}$ are the localization-union and $\mathrm{U}_{\mathrm{e} 3}, \mathrm{U}_{\mathrm{t} 3}$ are two estimate positions. If $\mathrm{A}_{2}$ is chosen as the verifier and the ranging error exists, the distance $d_{e 3}$ and $d_{13}$ won't distinguish widely because $A_{2}$ is almost in line with the nodes $\mathrm{A}_{3}$ and $\mathrm{A}_{4}$. In this situation, we will possibly get $\operatorname{Err}_{\mathrm{t} 1}>\mathrm{Err}_{\mathrm{e} 1}$ and take $\mathrm{U}_{\mathrm{e} 3}$ as the real estimate position of the $\mathrm{UN}$, which is not the correct decision. 


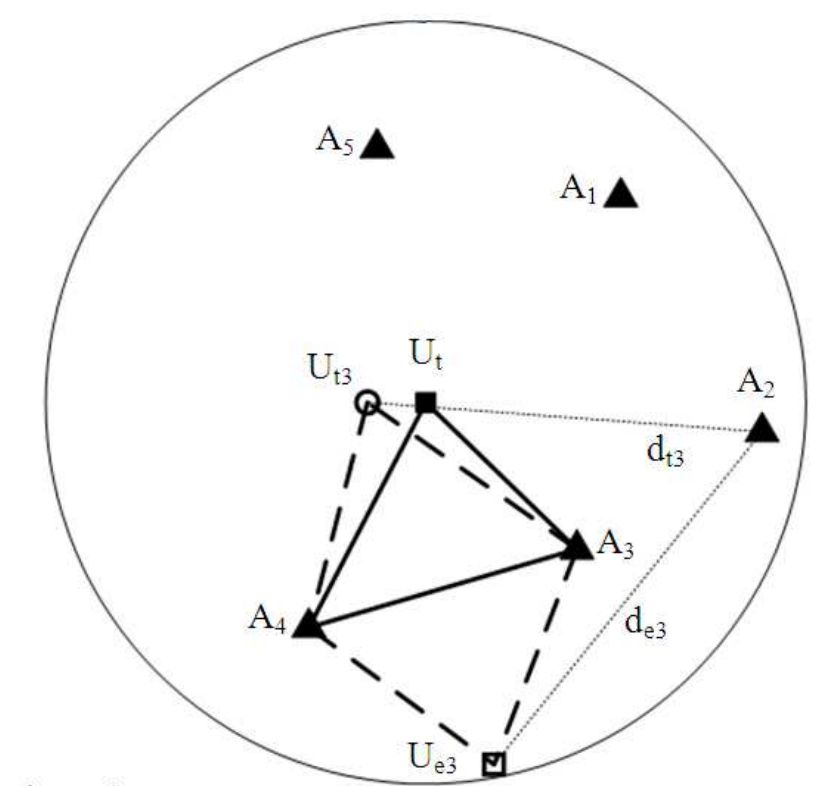

$\Delta$ Ancho $\square$ UNOEstimate location $\square$ Pseudo location

Fig. 6. Special case of verification method
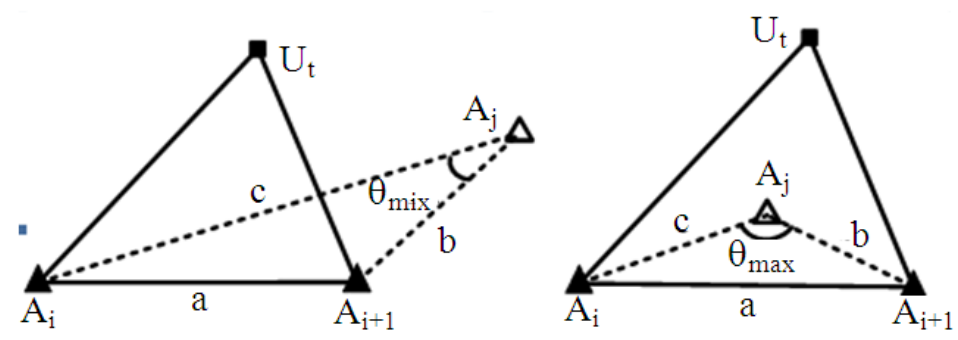

\section{$\Delta$ Anchor $\Delta \mathrm{VN}$}

Fig. 7. Selection of verifying node

To overcome the above problem, a selection scheme is proposed for the verifying node. As shown in Fig. 7, $A_{i}$ and $A_{t+1}$ make up of a localization-union and $A_{j}$ is taken as the verifier, then we have the angle $\theta$ which is formed by the three nodes $\left(A_{j}, A_{i}\right.$ and $\left.A_{i+1}\right)$ Equation (13):

$$
\theta=\arccos \left(\frac{b^{2}+c^{2}-a^{2}}{2 \times b \times c}\right)
$$

As we all known, $0 \leq \theta \leq \pi$ and $\theta$ affects the verification if it is too small or too big. Considering the above situation, we can choose the angle threshold $\theta\left(\theta_{\min } \leq \theta \leq \theta_{\max }\right)$.
However, the angle can't be measured directly for the range-only sensors. To circumvent this issue, an estimate scheme for relative-angle between nodes is proposed in the next subsection.

\subsubsection{Estimate Scheme for Relative-Angle Between Nodes}

Angle of Arrival (AOA) is a traditional angle directly-measure method for sensors but it is energyconsuming and needs else equipments. Moreover, the performance of AOA is affected by lots of environment factors such as multipath propagation. To solve the angle-measure problem in this study, an 
estimate algorithm of relative angle between nodes is proposed herein.

\section{Definition 3:}

Pseudo Node $\mathrm{U}_{\mathrm{c}}\left(\mathrm{x}_{\mathrm{o}}, \mathrm{y}_{\mathrm{o}}\right)$.

Assume that there are $\mathrm{N}$ anchors $\mathrm{A}_{\mathrm{i}}\left(\mathrm{x}_{\mathrm{i}}, \mathrm{y}_{\mathrm{i}}\right)$ in the communication range of a $U N$ named as $U$ and the distance between each anchor and the UN is denoted by $d_{i} i=1,2, \ldots, N$, then the pseudo node of the UN is defined as the weighted centroid of the $\mathrm{N}$ anchors and its position can be calculated by Equation (14):

$$
\left(x_{o}, y_{o}\right)=\left(\left(\sum_{i=1}^{N} \frac{x_{i}}{d_{i}}\right) / N \times \sum_{i=1}^{N} \frac{1}{d_{i}},\left(\sum_{i=1}^{N}{\frac{y_{i}}{d_{i}}}^{d_{i}}\right) / N \times \sum_{i=1}^{N} \frac{1}{d_{i}}\right)
$$

\section{Definition 4:}

Polar Coordinate System (PCS) in the communication range of a UN.

As shown in Fig. 8, the position of $U$ is taken as the pole location; a radial $U_{c} L_{r}$ formed by $U$ and its nearest anchor $\mathrm{A}_{\mathrm{m}}$ is considered as the polar axis; the anticlockwise is defined as the positive direction. Then, for the any point $A_{i}$ in the communication range of $U, d_{i}$ denotes the length of $\mathrm{UA}_{i} ; \alpha_{i}$ represents the angle from $\mathrm{UL}$ to $\mathrm{UA}_{\mathrm{i}} ; \mathrm{d}_{\mathrm{i}}$ is the radius vector and $\alpha_{\mathrm{i}}$ is the polar angle with respect to the point; then the ordered pair $\left(\mathrm{d}_{\mathrm{i}}\right.$, $\alpha_{i}$ ) is called as the polar coordinate of $A_{i}$ and this coordinate system is called as the polar coordinate system in the communication range of the UN.

The above PCS is established using the knowledge of the actual position of the UN, which is not accessible before node localization step. All the information is that the neighbor anchors in the communication range area of the UN. Then, there is another problem that how to establish a coordinate system which is convenient for angle estimate between nodes.

To solve the above issue, the information about anchors received by the UN is taken full advantage of in DRBLA. The pseudo node Uc of a UN is estimated and will replace the UN to establish a Virtual Polar Coordinate System (VPCS), which will be specified as follows.

\section{Definition 5:}

VPCS in the communication range area of a UN.

As shown in Fig. 8, the position of Uc is taken as the pole location; a radial $\mathrm{U}_{\mathrm{c}} \mathrm{L}_{\mathrm{r}}$ formed by $\mathrm{Uc}$ and its nearest anchor $\mathrm{A}_{\mathrm{m}}$ is considered as the polar axis; and also, the anticlockwise is defined as the positive direction. Then, for the any point $A_{i}$ in the communication range of $U, d_{i r}$ denotes the length of $U_{c} A_{i} ; \alpha_{i r}$ represents the angle from
$U_{c} L_{r}$ to $U_{c} A_{i} ; d_{i r}$ is the radius vector and $\alpha_{i r}$ is the polar angle with respect to the point; then the ordered pair $\left(\mathrm{d}_{\mathrm{ir}}\right.$, $\alpha_{i r}$ ) is called as the virtual polar coordinate of $A_{i}$ and this coordinate system is called as the VPCS in the communication range area of the UN. Moreover, we have $\mathrm{d}_{\mathrm{ir}} \approx \mathrm{d}_{\mathrm{i}} \in[0, \mathrm{R}], \alpha_{\mathrm{ir}} \approx \alpha_{\mathrm{I}} \in[0,2 \pi]$.

Until now, the VPCS of a UN is established and can be used to estimate the angle between the radius (formed by each anchor and the pole point) and the polar axis. Specifically, the included angle $\left(\alpha_{i r}\right)$ between any two anchors ( $\mathrm{Ai}$ and $\mathrm{Am}$ ) and the pseudo point (Uc) can be calculated and is used to select suitable localization-union and verifier which is mentioned in the above subsections.

\subsubsection{Rough Location Estimated by Different Localization-Unions}

As shown in Fig. 1, there are $\mathrm{N}$ anchors in the communication range area of the UN and their location is denoted as $A_{i}\left(x_{i}, y_{i}\right)$, where $i=1,2, \ldots, N$. The distance between $U$ and each anchor is measured by RSS ranging model and denoted as $d_{i}$, then the pseudo location of $U$ can be calculated and is denoted as $\mathrm{U}_{\mathrm{c}}\left(\mathrm{x}_{\mathrm{o}}, \mathrm{y}_{\mathrm{o}}\right)$, which is used to establish a VPCS. Until now, the localization information accumulated in the memory of $U$ can be denoted as a matrix as follows Equation (15):

Data $=\left[\begin{array}{cccc}1 & \left(\mathrm{x}_{1}, \mathrm{y}_{1}\right) & \mathrm{d}_{1} & \alpha_{1} \\ 2 & \left(\mathrm{x}_{2}, \mathrm{y}_{2}\right) & \mathrm{d}_{2} & \alpha_{2} \\ \vdots & \cdots & \cdots & \vdots \\ \mathrm{N} & \left(\mathrm{x}_{\mathrm{N}}, \mathrm{y}_{\mathrm{N}}\right) & \mathrm{d}_{\mathrm{N}} & \alpha_{\mathrm{N}}\end{array}\right]$

Any two anchors $\left(A_{i}\right.$ and $\left.A_{j}\right)$ will be selected as a localization-union if the below range and angle test are satisfied Equation (16):

$\left\{\begin{array}{l}\left|d_{i}+d_{j}\right| \geq \sqrt{\left(x_{i}-x_{j}\right)^{2}+\left(y_{i}-y_{j}\right)^{2}} \\ \left|d_{i}-d_{j}\right| \leq \sqrt{\left(x_{i}-x_{j}\right)^{2}+\left(y_{i}-y_{j}\right)^{2}} \\ \pi / 2-\lambda_{\alpha} \leq\left|\alpha_{i}-\alpha_{j}\right| \leq \pi / 2+\lambda_{\alpha}\end{array}\right.$

Then, we have localization equations as follows Equation (17):

$\left\{\begin{array}{l}\left(x_{i j}^{e}-x_{i}\right)^{2}+\left(y_{i j}^{e}-y_{i}\right)^{2}=d_{i}^{2} \\ \left(x_{i j}^{e}-x_{j}\right)^{2}+\left(y_{i j}^{e}-y_{j}\right)^{2}=d_{j}^{2}\end{array}\right.$

where, $\left(x_{i j}^{e}, y_{i j}^{e}\right)$ represent the estimate location of the localization-union $\left(A_{i}\right.$ and $\left.A_{j}\right)$. 


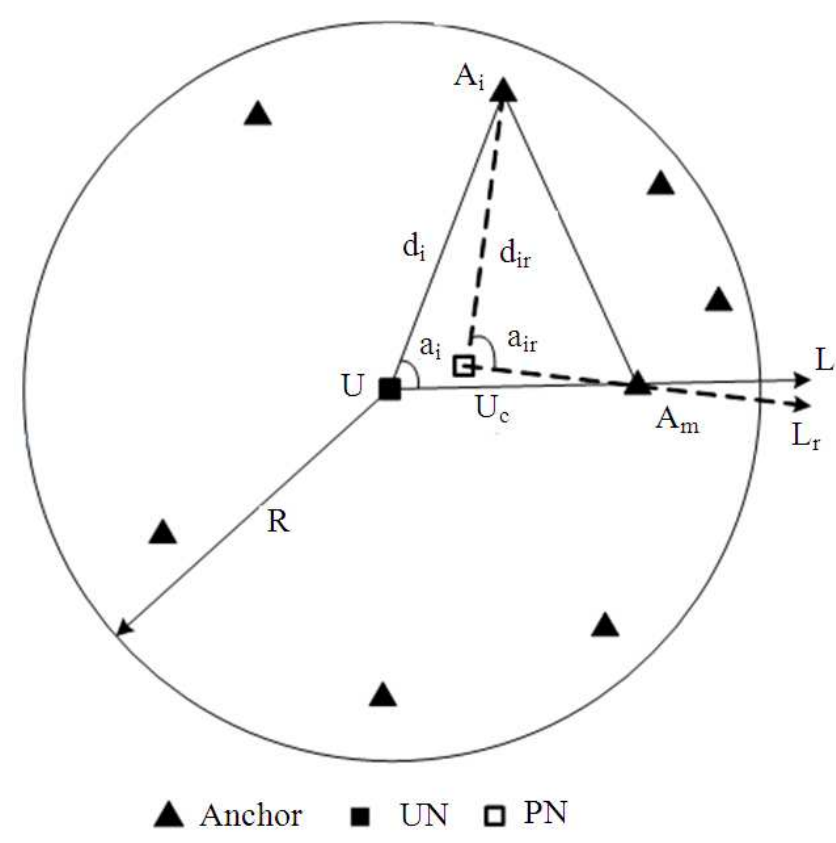

Fig. 8. Polar coordinate system and virtual polar coordinate

Here, another problem will come out as the number of anchors increase quickly. Considering that the increase of localization-unions will bring huge energy consuming and computation consuming but not obvious improvement of accuracy, a threshold named $\mathrm{N}_{\max }$ is designed in DRBLA. Specifically, if the number of received anchors is more than $\mathrm{N}_{\max }$, the anchor will take part in the range-angle test only if its RSS ranks in the top- $\mathrm{N}_{\max }$. This method may not only cut the consuming down but also improve the localization accuracy because greater RSS means more precise range measurement which is beneficial to node localization.

Another anchor $A_{k}\left(x_{k}, y_{k}\right)$ is employed as a verifier when there are two solutions for the localization-union and the verifier should satisfies the following angle test Equation (18):

$\theta_{\min } \leq \theta \leq \theta_{\max }$

where, $\theta$ is mentioned in Equation (13).

In this way, every localization-union can achieve a estimate location $\mathrm{U}_{\mathrm{tm}}\left(\mathrm{x}_{\mathrm{tm}}, \mathrm{y}_{\mathrm{tm}}\right)$ of the $\mathrm{UN}$ after the node verification step, where $\mathrm{m}=1,2, \ldots, \mathrm{M}$ means that there are $\mathrm{M}$ localization-unions.

\subsubsection{Refinement}

We can conclude from the Data matrix that different localization-unions achieve different estimate locations of the UN and these estimates contribute differently to the final estimate result of the UN. For more analyses, the distance between the UN and each anchor is different but the RSS ranging model has the property that close distance is relatively accurate and far distance is relatively fuzzy. Thus, the anchors in a localizationunion should contribute differently to the calculation of the final estimation of the node position. In DRBLA, the method of weighted mean is employed and the different rough estimates are combined to the final estimate value. The corresponding weight is given by Equation (19):

$$
\mathrm{W}_{\mathrm{tm}}=\frac{1}{\left|\mathrm{~d}_{\mathrm{im}}^{2}-\mathrm{d}_{\mathrm{jm}}^{2}\right|} / \sum_{\mathrm{m}=1 \mid}^{\mathrm{M} \mid} \frac{1}{\left|\mathrm{~d}_{\mathrm{m}}^{2}-\mathrm{d}_{\mathrm{jm}}^{2}\right|}
$$

where, $d_{i m}, d_{j m}$ denote the distance between anchor $A_{i}, A_{j}$ and the UN, respectively. Finally, we can achieve the final location estimate of the UN by Equation (20):

$\mathrm{x}_{\mathrm{e}}=\frac{\left(\sum_{\mathrm{m}=1}^{\mathrm{M}} \mathrm{w}_{\mathrm{tm}} \mathrm{x}_{\mathrm{tm}}\right)}{\sum_{\mathrm{m}=1}^{\mathrm{M}} \mathrm{w}_{\mathrm{tm}}}, \mathrm{y}_{\mathrm{e}}=\frac{\left(\sum_{\mathrm{m}=1}^{\mathrm{M}} \mathrm{w}_{\mathrm{tm}} \mathrm{y}_{\mathrm{tm}}\right)}{\sum_{\mathrm{m}=1}^{\mathrm{M}} \mathrm{w}_{\mathrm{tm}}}$

For the reason that Data matrix is updated periodically, the anchors which are broken-down because of energy- 
exhaustion will be detected and abandoned in the localization step. Thus, the robustness of DRBLA will be guaranteed because a small quantity of broken-down anchors won't affect the localization badly.

\subsubsection{DRBLA Overview}

The proposed DRBLA can be summarized as follows:

Step1: Initiation after node deployment

Step2: Anchors broadcast beacon information

Step3: UNs receive beacon information and calculate the distance between self and neighbor anchors according to RSS ranging model in (5)

Step4: Process according to the number $(\mathrm{N})$ of anchors received by a $\mathrm{UN}$

If $\mathrm{N}=0$, then the $\mathrm{UN}$ is unable to be localized and the localization is over

Else if $0<\mathrm{N}<3$, then take the pseudo location as the

estimate of the UN as shown in (14)

Else if $3 \leq \mathrm{N} \leq \mathrm{N}_{\max }$, break and go to step 5;

Else if $\mathrm{N} \geq \mathrm{N}_{\max }$, pick out these anchors whose RSS rank in the top- $\mathrm{N}_{\max }$, then break and go to step 5 End if

Step5: Calculate the coordinate of the pseudo node and establish the VPCS, then achieve the relative angles between different anchors

Step6: Choose some suitable anchors to form localization-unions according to (16) and select verifier to get the rough estimate of the UN according to (18)

Step7: Computer the weight corresponding to different rough estimate according to (19) and get the final fusion result according to (20)

\subsection{Numerical Illustration}

\subsubsection{Simulation Setting}

Without loss of generality, the unit of range parameter is set to be meter and is denoted as $\mathrm{m}$ :

- Experiment scenario: All nodes are deployed in a $100 \times 100 \mathrm{~m}$ size standard square area and the max communication range of all nodes is $\mathrm{R}$, which can also be used to adjust the density of anchors in the network

- Average Anchor Heard (AAH) represents the average number of anchors received by each node in the network

- Degree of noise (DON) is defined as the standard deviation $(\sigma)$ of a Gauss distribution with zero-mean and denotes the level of noise. Figure 9 (a), (b) show the radio propagation when DON $=0.1$ and DON $=0.2$, respectively

- Average Localization Error (ALE) is defined as Equation (21):

$$
\mathrm{ALE}=\frac{\sum_{\mathrm{i}=1}^{\mathrm{N}_{1}} \sqrt{\left(\mathrm{x}_{\mathrm{ei}}-\mathrm{x}_{\mathrm{ai}}\right)^{2}+\left(\mathrm{y}_{\mathrm{ei}}-\mathrm{y}_{\mathrm{ai}}\right)^{2}}}{\mathrm{~N}_{1} \times \mathrm{R}}
$$

where, $\mathrm{N}_{\mathrm{l}}$ denotes the number of localized UNs and $\left(\mathrm{x}_{\mathrm{ai}}, \mathrm{y}_{\mathrm{ai}}\right)$ is the actual position of the corresponding UNs while $\left(\mathrm{x}_{\mathrm{ei}}, \mathrm{y}_{\mathrm{ei}}\right)$ is the estimate position.

- Communication Overhead (CO) is defined as the average Number of Messages (NMs) broadcasted in the whole network

In this simulation, other important parameters is set to be $\lambda_{\alpha}=\pi / 6, \theta_{\operatorname{man}}=5 \pi / 6$ and $\theta_{\min }=\pi / 6$. For a fair evaluation, we made a total of $\mathrm{N}=100$ independent Monte Carlo runs using MATLAB.

\subsubsection{ALE Varying AAH}

When DON $=0.1$, Fig. 10 and 11 indicate the ALE varying AAH when nodes are deployed uniformly and randomly, respectively.

As shown in the Fig. 10 and 11, ALE of the three algorithms decreases as AAH increase because the more useful information utilized in the node localization as more anchors deployed in the network. Obviously, DRBLA outperform the other two algorithms at all the point of AAH no matter nodes are deployed uniformly or randomly for the reason that DRBLA has taken more advantage of anchor information and employed some effective scheme like localization-union configuration and verification. However, when AAH is close to 20, ALE of the three algorithms converge gradually. The unsolvable localization error is mostly caused by other environment factors like multipath propagation and the increase of AAH can't essentially solve the bad effect brought by ranging error.

In addition, in the aspect of localization scheme, WCentroid mainly use the method of weighted mean and is over dependent on the regular deployment of anchors; RSSI-MLE can achieve equal localization accuracy by multiple iteration but this method will definitely arise the time-consuming and energy-consuming. 


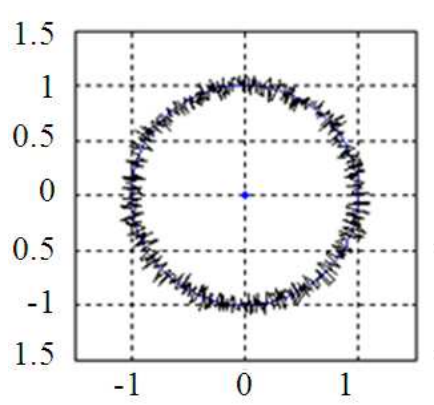

(a)

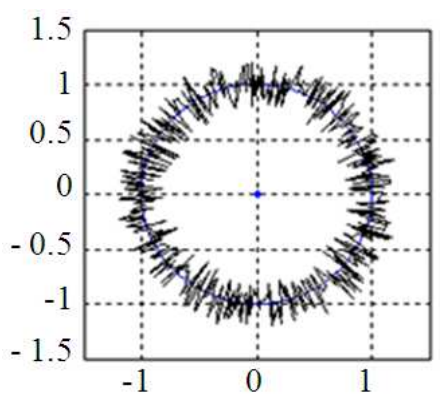

(b)

Fig. 9. Radio propagation pattern when $\mathrm{DON}$ exists, (a) $\mathrm{DON}=0.1$, (b) $\mathrm{DON}=0.2$

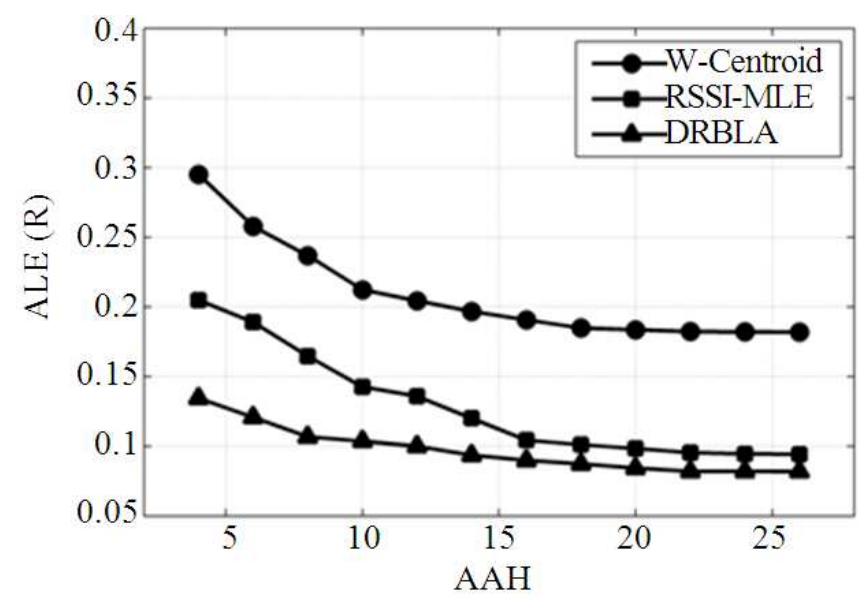

Fig. 10. ALE varying AAH, DON $=0.1$, Uniform

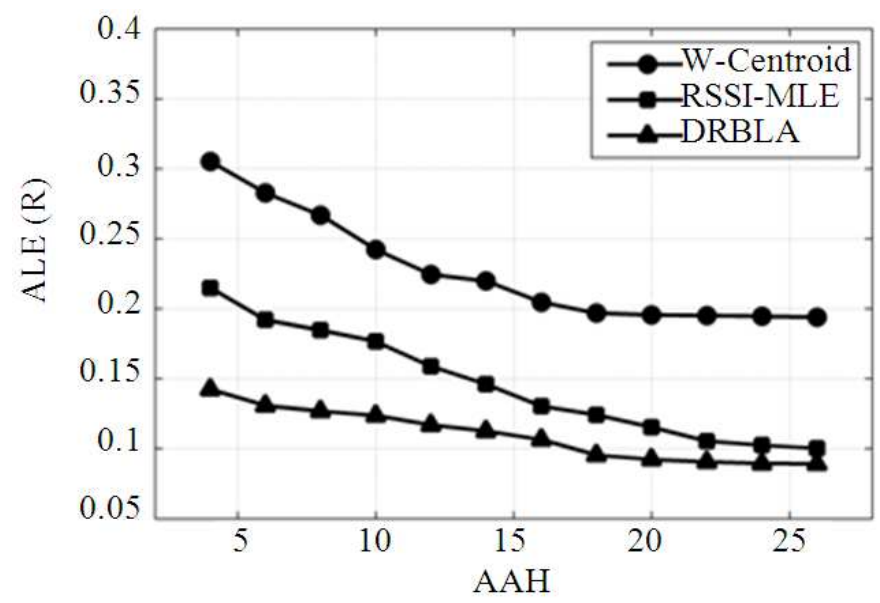

Fig. 11. ALE varying AAH, DON = 0.1, Random

However, the effect of topology of anchors to node localization is taken into account in DRBLA and range-angle information has been employed effectively to improve estimate accuracy. Specifically, 
if DON $=0.1$, the ALE of DRBLA can be guaranteed below $15 \%$ if only $\mathrm{AAH} \geq 3$. The divide-and-conquer and refinement method are key factors for improving the localization accuracy.

Specially, when $\mathrm{AAH}=4$ and nodes are deployed uniformly, the simulation results show that DRBLA improve the localization accuracy in $\sim 16 \%$ of WCentroid and $\sim 8 \%$ of RSSI-MLE. For the randomly deployment case, DRBLA reduces the localization error in $\sim 17 \%$ of $\mathrm{W}$-Centroid and $\sim 9 \%$ of RSSI-MLE. These results indicate the distinct advantage of DRBLA in the sparse-anchors network.

\subsubsection{ALE Varying DON}

When $A A H=10$, Fig. 12 and 13 indicate the ALE varying DON when nodes are deployed uniformly and randomly, respectively.

As shown in Fig. 12 and 13, DON affects ALE severely and it means that the ranging error caused by signal interference brings negative effect on localization. As a whole, the average improvement on ALE of DRBLA is about $10 \%-12 \%$ compared with W-Centroid and about 4\%-6\% compared with RSSI-MLE. Specially, the ALE of DRBLA is below $30 \%$ if only DON $\leq 0.3$, which meets most applications.

\subsubsection{CO Varying AAH}

Figure 14 indicates the average $\mathrm{CO}$ of the three algorithms mentioned in this study varying $\mathrm{AAH}$ when nodes are deployed in a $100 \mathrm{~m} \times 100 \mathrm{~m}$ size standard square area.

In theory, anchors need to broadcast the beacon messages once and UNs can realize localization if only messages are received in the three algorithms. Compared with W-Centroid and RSSI-MLE, DRBLA improves the localization accuracy by utilizing the relation between anchors and UNs and it doesn't cause additional CO, which is showed in Fig. 14.

\subsubsection{Complexity Analyses}

Obviously, the computation complexity of DRBLA is $\mathrm{O}(\mathrm{M})$, where $\mathrm{M}$ is the number of localization-unions. It only needs several matrix addition and multiplication in the calculation of a rough estimate. On the other side, a threshold $\mathrm{N}_{\max }$ is set in DRBLA to avoid the infinite increase of computation caused by the increase of AAH.

On the whole, DRBLA is a distributed hybrid localization algorithm which does not need any additional equipment like AOA and the UNs can realize easy and effective localization after receiving beacon messages in the network and then, the CO of DRBLA is acceptable because only anchors in the network need to broadcast messages once and the UNs only receive messages. Moreover, the robustness of DRBLA can be guaranteed because anchors update their neighbor data periodically so that some wrong or broken-down anchors will be detected in time and abandoned in the node localization step.

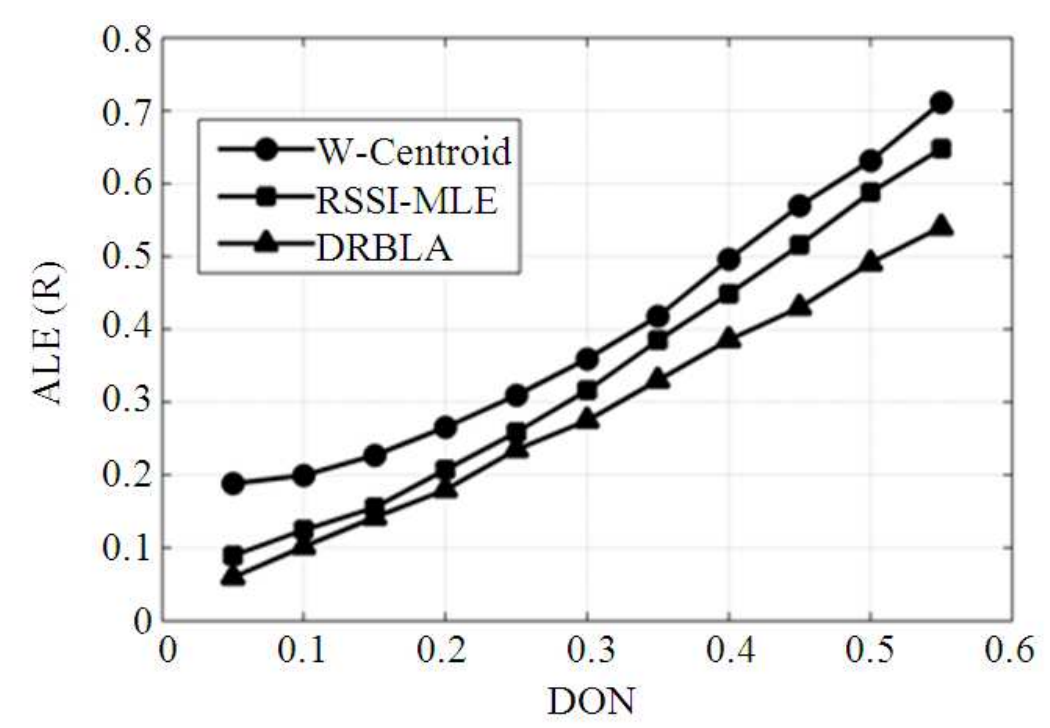

Fig. 12. ALE varying DON, AAH = 10, Uniform 


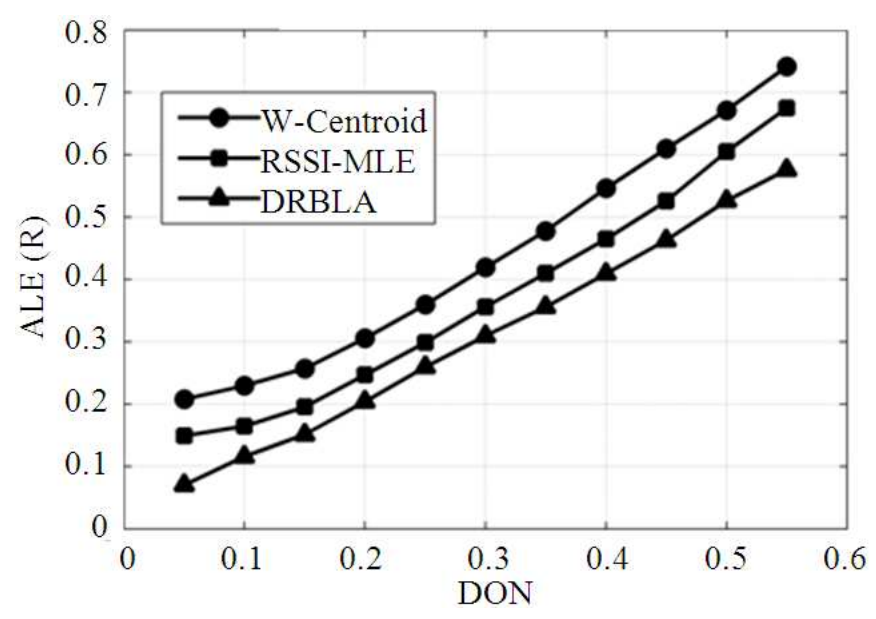

Fig. 13. ALE varying DON, AAH=10, Random

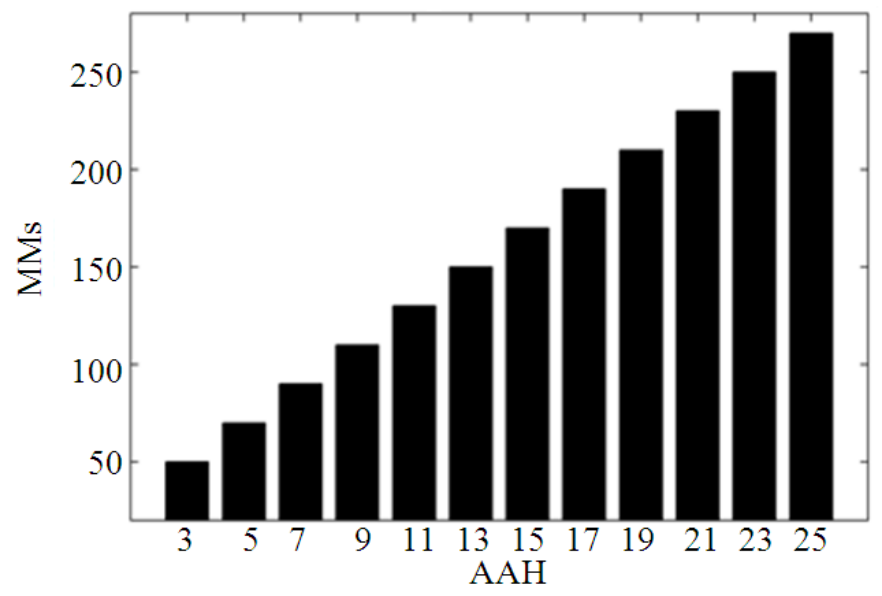

Fig. 14. $\mathrm{CO}$ varying $\mathrm{AAH}$

\section{CONCLUSION}

In this note, a distributed node localization algorithm based on divide-and-conquer and refinement method is proposed and simulated. First, RSS is translated into distance and anchors are seriated in clockwise order. Then, effective anchors are selected out orderly to estimate coordinate of unknown node and a verifying node is selected out to identify the estimate coordinate. Finally, the weighted centroid of all the cursory coordinates is identified as the location result. No added hardware is needed in this algorithm and communication spending is very low. Our work is compared with other traditional localization schemes and simulation result indicates the effectiveness of DRBLA whether anchors are placed regularly or randomly. Especially, it can get high localization accuracy with low anchor density.

\section{ACKNOWLEDGEMENT}

This study was financially supported by National Natural Science Foundation of China under grant number 61032001 and Shandong Provincial Natural Science Foundation of China under grant number ZR2012FQ004.

\section{REFERENCES}

Amitangshu, P., 2010. Localization algorithms in wireless sensor networks: Current approaches and future challenges. Netw. Protocols Algorithms, 2: 45-74. DOI: 10.5296/npa.v2i1.279

Barani, H. and M. Fathy, 2010. An algorithm for localization in vehicular ad-hoc networks. J. Comput. Sci., 6: 168-172. DOI: 10.3844/jcssp.2010.168.172 
Blumenthal, J., R. Grossmann, F. Golatowski and D. Timmermann, 2007. Weighted centroid localization in zigbee-based sensor networks. Proceedings of the IEEE International Symposium on Intelligent Signal Processing, Oct. 3-5, IEEE Xplore Press, Alcala de Henares, $\quad$ pp: 1-6. DOI: 10.1109/WISP.2007.4447528

Bulusu, N., J. Heidemann and D. Estrin, 2000. GPS-less low-cost outdoor localization for very small devices. IEEE Personal Commun. Mag., 7: 28-34. DOI: $10.1109 / 98.878533$

He, T., C. Huang, B.M. Blum, J.A. Stankovic and T. Abdelzaher, 2003. Range-free localization schemes for large scale sensor networks. Proceedings of the 9th Annual International Conference on Mobile Computing and Networking, Sept. 14-19, ACM Press, New York, USA., pp: 81-95. DOI: 10.1145/938985.938995

Iskandarani, M.Z., 2011. A novel approach to head positioning using fixed center interpolation net with weight elimination algorithm. J. Comput. Sci., 7: 173-178. DOI: 10.3844/jessp.2011.173.178

Jauregui-Ortiz, S., M. Siller and F. Ramos, 2011. Node localization in WSN using trigonometric figures. Proceedings of the IEEE Topical Conference on Wireless Sensors and Sensor Networks (WiSNet), Jan. 16-19, IEEE Xplore Press, Phoenix, AZ., pp: 65-68. DOI: 10.1109/WISNET.2011.5725030

Li, R.Z., X.L. Luo and J.R. Lin, 2011. Weighted centroid correction localization in cellular systems. Am. J. Eng. Applied Sci., 4: 37-41. DOI: 10.3844/ajeassp.2011.37.41
Liu, J., 2009. Research on range-based localization algorithm of wireless sensor networks. Aeronautical Comput. Technique, 39: 123-126.

Liu, Y., X. Yi and Y. He, 2012. A novel centroid localization for wireless sensor networks. Int. J. Distrib. Sensor Netw., 2012: 8-8. DOI: 10.1155/2012/ 829253

Newlin, D.R. and E.C. Monie, 2013. Edge sensing demosaicing using adaptive weighted interpolation. Am. J. Applied Sci., 10: 418-425. DOI: 10.3844/ajassp.2013.418.425

Shi, Q.J., C. He and H.Y. Chen, 2010. Distributed wireless sensor network localization via sequential greedy optimization algorithm. IEEE Trans. Signal Process., 58: 3328-3340. DOI: 10.1186/1687-14992012-152.

Vera, R., S. F. Ochoa and R.G. Aldunate, 2011. EDIPS: An easy to deploy indoor positioning system to support loosely coupled mobile work. J. Personal Ubiqui. Comput., 15: 365-376. DOI: 10.1007/s00779-010-0357-X

Wang, F.B., L. Shi and F.Y. Ren, 2005. SelfLocalization systems and algorithms for wireless sensor networks. J. Soft., 16: 857-868. DOI: $10.1360 /$ jos 160857

Zhou, Y., 2009. Study of wireless sensor network localization algorithm based on RSSI. Comput. Sci., 36: 119-121. 